\title{
TAURUS observations of active galaxies: NGC 1275 and NGC 4151
}

\author{
S.W.Unger ${ }^{1}$, K.Taylor ${ }^{2}$, A.Pedlar ${ }^{3}$, H.Ghataure ${ }^{3}$, M.V.Penston ${ }^{1}$ \& D.J.Axon ${ }^{3}$. \\ 1) Royal Greenwich Observatory, Herstmonceux Castle, E.Sussex BN27 1RP, UK. \\ 2) Anglo-Australian Observatory, PO Box 296, Epping, NSW 2121, Australia. \\ 3) Jodrell Bank, Macclesfield, Cheshire SK11 9DL, UK.
}

ABSTRACT. We have used the TAURUS technique to study the morphology and velocity field of spatially extended emission-line gas in a number of active galaxies. This paper describes our results for NGC 1275 and NGC 4151. The emission line maps of NGC 1275 are the first scientific data from the new 4.2-m William Herschel Telescope, and show that the morphology of the foreground galaxy falling into the Perseus cluster bears a close relationship to that of NGC 1275 itself, implying that the two galaxies are physically associated.

In the case of NGC 4151 , we find the emission line region is elongated along the same axis as the nuclear radio source. This cannot be due to emission-line gas being accelerated by radio ejecta, since the emission-line gas rotates with the galactic disk. We suggest that the emission-line gas is being ionized by a highly anisotropic nuclear continuum source, UV photons escaping from the nucleus preferentially along the radio axis.

\section{Introduction}

TAURUS is a wavelength-scanned imaging Fabry-Perot interferometer, which produces spectra on a fully-sampled grid of points covering an extended emission line object. The original version of the instrument was built at the Royal Greenwich Observatory in collaboration with Imperial College London, and is described in detail by Taylor \& Atherton (1980) and Atherton et al. (1982). It is currently in operation on the Isaac Newton Telescope. The success of the TAURUS concept stimulated interest in creating a fully engineered common-user version of the system. As part of the UK/Dutch collaboration on the Isaac Newton group of telescopes, the Kapteyn Sterrenwacht at Roden, NL took on the design and manufacturing task, and TAURUS Mk 2 was commissioned as the first instrument on the newly completed $4.2-\mathrm{m}$ William Herschel Telescope in August 1987.

The complete $2 \mathrm{D}$ coverage provided by TAURUS makes this instrument extremely well-suited for studying the complex velocity fields and morphologies of spatially extended emission line regions in active galaxies. In this paper we describe observations of two rather different types of emission line region, the high velocity gas in NGC 1275 and the Extended Narrow Line Region in NGC 4151.

\section{The nature of the high velocity gas in Perseus $A$}

NGC 1275, the central galaxy of the Perseus cluster, has presented an intriguing puzzle ever since Minkowski (1957) discovered two discrete systems of emission line gas at redshifts of 5300 and $8200 \mathrm{~km} / \mathrm{s}$.

The low velocity system is at the same redshift as the galaxy. Narrow-band $\mathrm{H} \alpha$ images show that this emission line gas is highly filamentary in nature (Lynds 1970). The filaments are 
embedded in an extensive $\mathrm{X}$-ray halo which may be accreting onto the galaxy, and it has been suggested that the filaments are a result of a thermal instability in this accretion flow (Fabian \& Nulsen 1977). NGC 1275 is also associated with one of the brightest extragalactic radio sources (Perseus $\mathrm{A} \equiv 3 \mathrm{C} 84$ ), and it may be that the formation of some group of optical filaments is related to the ejection of radio-emitting plasma (Pedlar et al. 1983)

The nature of the high velocity system is even less well understood. The high velocity gas is seen in absorption against NGC 1275 and so in spite of its much higher redshift must represent foreground material (de Young et al. 1973). The available data on the kinematics and excitation of the gas suggest a picture in which the high velocity system is a spiral galaxy falling into the centre of the Perseus cluster with a relative velocity of $3000 \mathrm{~km} / \mathrm{s}$ (Rubin et al. 1977; Kent \& Sargent 1979)

Because of the problem associated with the survival of the gaseous component of a galaxy as it passes through the $\mathrm{X}$-ray halo, most authors have assumed that the two filamentary systems are physically unrelated and appeal to spatial coincidence in alignment to explain the proximity of the two systems. However Hu et al (1985), who present the best flux map of the low velocity system to date, argue for an alternative view suggesting that the high velocity system is feeding an accretion flow which reveals itself as the low velocity gas.

We have used TAURUS observations to derive the morphology and velocity field of both emission line systems. Observations of the low velocity system were taken on the 2nd December 1986 at the Isaac Newton Telescope using the original version of the TAURUS instrument. The red-shifted $\mathrm{H} \alpha$ line was observed with a velocity resolution of $45 \mathrm{~km} / \mathrm{s}$, a free spectral range of $1200 \mathrm{~km} / \mathrm{s}$ and a spatial scale of $0.7 \mathrm{arcsec} /$ pixel. Observations of the high velocity system were taken on the 21st August 1987 at the William Herschel Telescope, as part of the commissioning tests for TAURUS Mk 2. The red-shifted [OIII] $\lambda 5007$ line was observed with a velocity resolution of about $30 \mathrm{~km} / \mathrm{s}$, a free spectral range of $575 \mathrm{~km} / \mathrm{s}$ and a spatial scale of $1.3 \mathrm{arcsec} / \mathrm{pixel}$. Both datasets were reduced using the methods described by Atherton et al. (1982) and Taylor (1984).

Fig. 1 shows as a grey-scaled image the spatial distribution of the high-velocity emission line gas, superimposed on a contour plot of the low-velocity emission line gas. The most striking feature of the morphology of the high velocity system is that most of the line emission is confined to two distinct regions, separated by 25 arcsec (about $8.5 \mathrm{kpc}$ ) in $\mathrm{PA}=110^{\circ}$. Both regions are slightly extended. The eastern region shows two 'tongues' of emission, one extending for 10 arcsec in $\mathrm{PA}=-3^{\circ}$, the other extending for about 15 arcsec to the east before bending abruptly through $90^{\circ}$ to continue northwards. The western region is elongated along $\mathrm{PA}=135^{\circ}$. Neither region is associated with the continuum nucleus of NGC 1275.

Unlike the high velocity system, the brightest peak of the low velocity system is centred on the continuum nucleus of NGC 1275. There are three other emission peaks, 5, 16 and 20 arcsec from the nucleus in PAs $-74^{\circ},-93^{\circ}$ and $57^{\circ}$ respectively. The peaks are embedded in more diffuse emission, which continues out into the large scale filamentary system.

It is clear from Fig. 1 that the two systems of emission-line gas have fairly similar linear extents, and are both roughly elongated along an east-west direction. Probably the most important result of our new observations is that in spite of this general similarity, the spatial distribution 


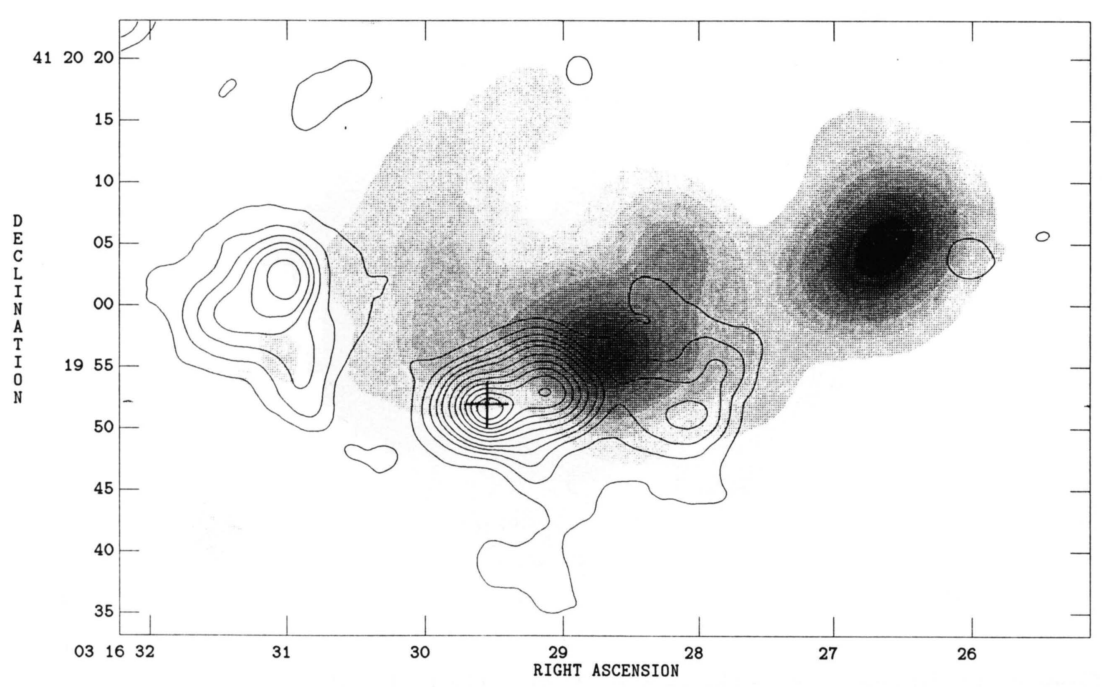

Fig. 1 Grey-scaled image showing the spatial distribution of the high-velocity emission line gas superimposed on a contour plot showing the spatial distribution of the low-velocity gas. The position of the continuum nucleus is marked with a cross.

of the high velocity gas is in fact anti-correlated with that of the low velocity gas. The four peaks in the map of the low velocity emission all lie on the southern or eastern edges of the emission from the eastern component of the high velocity system.

The velocity field of the high velocity gas (Fig. 2) is also rather more complex than suggested by previous observations. To the east, our observations are consistent with earlier data, which suggested that the velocity varies smoothly from east to west, with the highest velocities to the west, as would be expected from a rotating edge-on galaxy. However, the velocity field of the western emission line region shows a completely different behaviour, with the velocity gradient in a roughly north-south direction. As noted by previous authors, the high velocity gas has a much narrower line-width than the low velocity gas, the lines being unresolved at the $30 \mathrm{~km} / \mathrm{s}$ resolution of our present data.

What does all this mean? It is clear that the high velocity system is not a foreground galaxy unrelated to NGC 1275, since the peculiar morphology and velocity field are not consistent with a normal undisturbed galaxy. The only plausible model we are left with is one in which the high velocity system represents a galaxy which has fallen into the centre of the Perseus cluster and is now in the process of colliding with NGC 1275. The peculiar morphology and velocity field of the high velocity system could probably be explained in such a nodel as being due to tidal effects between the closely interacting galaxies. The anti-correlation between the two emission line systems might also be expected in such a model, since where the two galaxies meet, at a relative velocity of about $3000 \mathrm{~km} / \mathrm{s}$, the emission-liur gas will be shock-heated to about $10^{8} \mathrm{~K}$, too hot to radiate optical emission lines. 


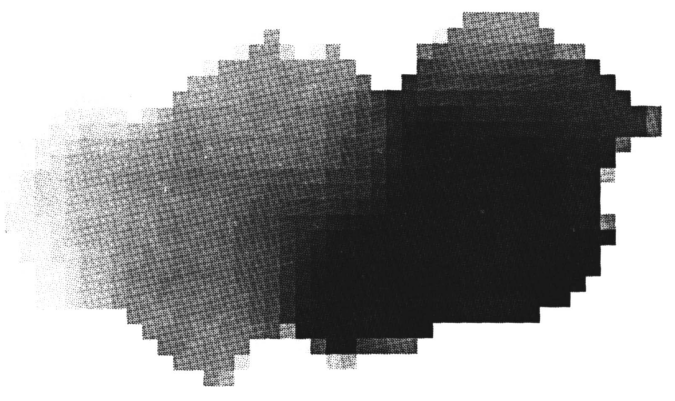

Fig. 2 Grey-scaled image showing the velocity field of the high-velocity emission line system in NGC 1275. The velocity range covered is $250 \mathrm{~km} / \mathrm{s}$, with higher recession velocities denoted by darker shading.

\section{The Extended Narrow Line Region in NGC 4151}

The high-excitation line emission from Seyfert nuclei is normally considered to be dominated by two regions, the Broad Line Region, with a scale size of order $0.1 \mathrm{pc}$, and the Narrow Line region, with a scale size of a few hundred parsecs. However, recent long-slit spectroscopy of a number of Seyfert galaxies has shown the presence of some high-excitation emission-line gas at much larger distances (up to $20 \mathrm{kpc}$ ) from the galactic nuclei (e.g. Unger et al. 1987). The emission lines from this 'Extended Narrow Line Region' (ENLR) have a narrow line-width (FWHM $<45 \mathrm{~km} / \mathrm{s}$ ) and a velocity field consistent with normal galactic rotation. The long-slit observations show that the ENLR is in general more extended along the axis of the nuclear radio source than perpendicular to it. However, we obviously need to map the spatial distribution of the ENLR more fully in order to quantify how good the alignment is between the ENLR and the radio axes.

We have therefore used TAURUS to map the velocity field and intensity distribution of the ENLR in NGC 4151. The observations were taken at the Isaac Newton Telescope on the 17th March 1986. The red-shifted [OIII] $\lambda 5007$ line was observed with a velocity resolution of about $30 \mathrm{~km} / \mathrm{s}$, a free spectral range of $970 \mathrm{~km} / \mathrm{s}$ and a spatial scale of $0.56 \mathrm{arcsec} / \mathrm{pixel}$.

Fig. 3 shows the morphology of the ENLR in NGC 4151 derived from these TAURUS observations. This is broadly consistent with previous narrow-band imaging observations (Heckman \& Balick 1983), which showed a narrow, knotty feature extending about $2 \mathrm{kpc}$ to the southwest of the nucleus together with a weaker protrusion extending about $0.5 \mathrm{kpc}$ to the northeast.

Fig. 3 also shows TAURUS spectra at four representative points: close to the nucleus; well away from the nucleus to the southwest and northeast; at an iut rmediate position to the southwest. This clearly shows the distinction between the relatively broad lines associated with 


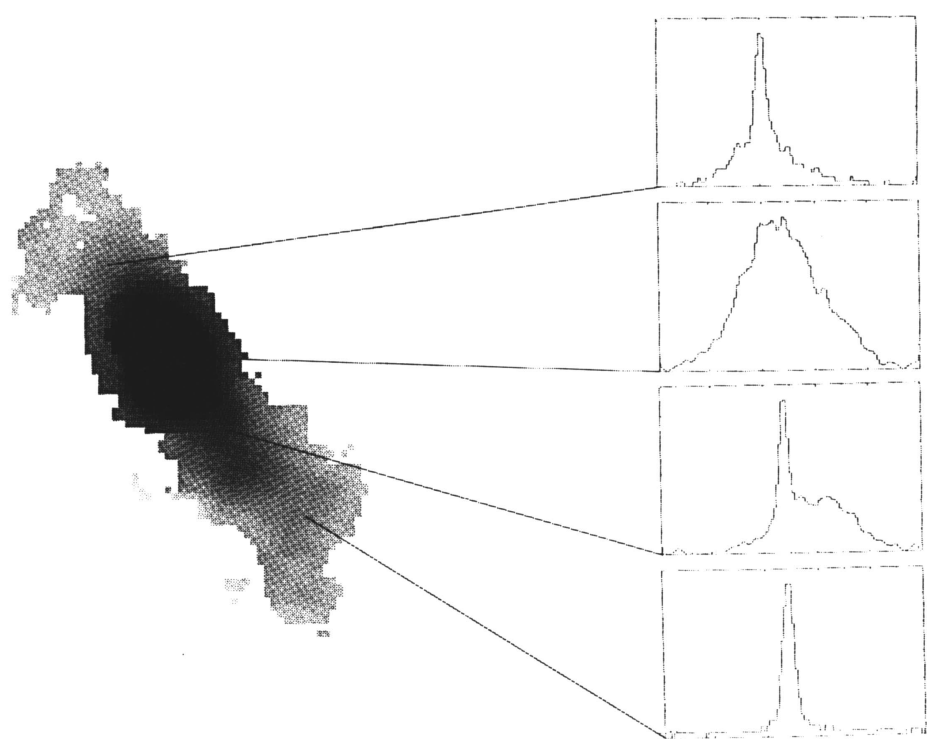

Fig. 3 The distribution of emission line gas in the nucleus of NGC 4151. Spectra are shown at four representative points, and cover a velocity range of $970 \mathrm{~km} / \mathrm{s}$ with a resolution of about $30 \mathrm{~km} / \mathrm{s}$. The spectra are plotted with wavelength decreasing towards the right.

the conventional Narrow Line Region close to the nucleus, and the unresolved lines from the ENLR. It can be seen from the spectrum at the intermediate position to the southwest that the ENLR does not simply represent a continuation of the NLR, with the line-width gradually getting narrower away from the nucleus. The ENLR is a distinct kinematic component from the NLR, and can be traced into the nucleus until the point where it is swamped by the NLR emission.

As we noted above, one of the most striking features about the ENLR is that it is highly elongated, along the same PA as the nuclear radio source (Harrison et al. 1986). This cannot be due to the emission line gas being ejected by the radio jet, since a comparison of the velocity field of the ENLR with VLA observations of the neutral hydrogen in the galactic disk shows that the ENLR rotates like the undisturbed disk gas (Fig. 4).

There is a striking similarity here with the high excitation gas in Centaurus A (Phillips et al. 1984) which, although having a completely different spatial morphology to that of the underlying low excitation disk, still shares in its rotation.

Unger et al. (1987) suggest that the alignment of the ENLR with the radio axis is due to the nuclear UV field which ionizes the ENLR being highly anisotropic, with UV photons escaping from the galactic nucleus preferentially along the radio axis. This model is supported by our new observations of NGC 4151. The idea of an anisotropic continumm nucleus is of course not new, and has been put forward to explain a number of properli... nctive galactic nuclei. The importance of our TAURUS observations is that, if our modei . "l the ENLR is correct, it is 


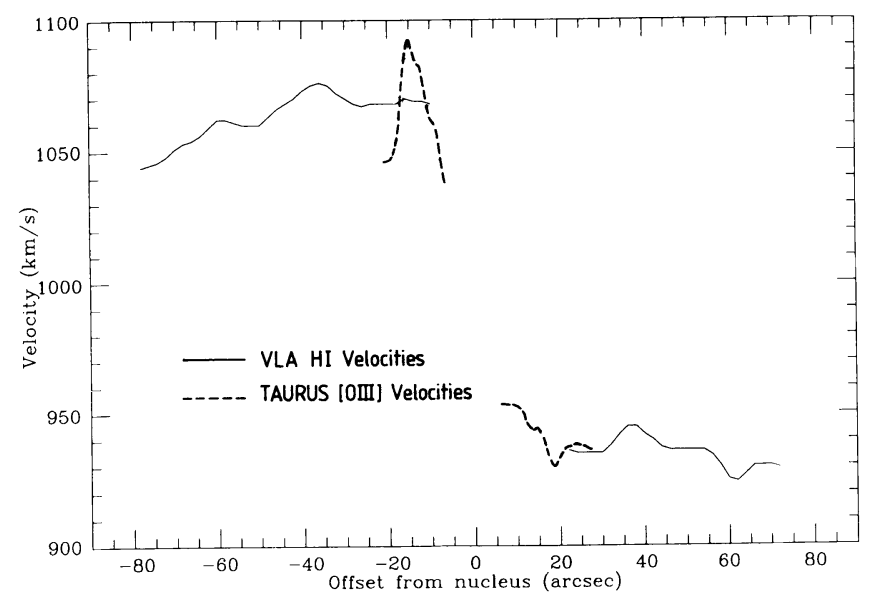

Fig. 4 Rotation curve of NGC 4151, derived from the TAURUS observations at [OIII] $\lambda 5007$ described in this paper and VLA neutral hydrogen observations (Pedlar et al. In preparation). Note the good agreement between the two rotation curves, implying that the high-excitation gas rotates with the galactic disk.

possible to determine the form of the anisotropy by mapping the spatial distribution of the ENLR gas. We find that the 5 brightest emission-line knots in the ENLR all lie within a cone of opening angle about $20^{\circ}$ as seen from the nucleus, implying that most of the ionizing flux from the continuum nucleus is confined to within a cone of the same opening angle.

\section{References}

Atherton, P.D., Taylor, K., Pike, C.D., Harmer, C.F.W., Parker, N.M. \& Hook, R.N., 1982. Mon.Not.R.astr.Soc., 201, 661.

de Young, D.S., Roberts, M.S. \& Saslaw, W.C., 1973. Astrophys.J., 185, 809.

Fabian, A.C. \& Nulsen, P.E.J., 1977. Mon.Not.R.astr.Soc., 180, 479.

Harrison, B., Pedlar, A., Unger, S.W., Burgess, P., Graham, D.A. \& Preuss, E., 1986. Mon.Not.R.astr.Soc., 218, 775.

Heckman, T.M. \& Balick, B., 1983. Astrophys.J., 268, 102.

Hu, E.M., Cowie, L.L., Kaaret, P., Jenkins, E.B., York, D.G. \& Roesler, F.L., 1983. Astrophys.J., 275, L27.

Kent, S.M. \& Sargent, W.L.W., 1979. Astrophys.J., 230, 667.

Lynds, C.R., 1970. Astrophys.J., 159, L151.

Minkowski, R., 1957. IAU Symposium No. 4, ed. H.C.van der Hulst, publ. CUP, p.107.

Pedlar, A., Booler, R.V. \& Davies, R.D., 1983. Mon.Not.R.astr.Soc., 203, 667.

Phillips, M.M., Taylor, K., Axon, D.J., Atherton, P.D. \& Hook, R.N., 1984 Nature, 310, 554.

Rubin, V.C., Ford, W.K., Peterson, C.J. \& Oort, J.H., 1977. Astrophys.J., 211, 693.

Taylor, K., 1984. Indirect Imaging, ed. J.A.Roberts, publ. CUP, p.379.

Taylor, K. \& Atherton, P.D., 1980. Mon.Not.R.astr.Soc., 191, 675.

Unger, S.W., Pedlar, A., Axon, D.J., Whittle, M., Meurs, E.J.A. \& Ward, M.J., 1987. Mon.Not.R.astr.Soc., 228, 671. 